Ann. Zootech., I972, 21 (I), 29-35.

\title{
INTÉRÊT DE LA PROPHYLAXIE DE LA MYOPATHIE DU VEAU PAR ADDITION DE SÉLÉNIUM AUX COMPLÉMENTS MINÉRAUX DES MÈRES
}

\author{
M. LAMAND \\ avec la collaboration technique de Claudine LaB et de P. Chagnavd \\ Station de Physiopathologie de la Nutrition, \\ Centre de Recherches de Clermont-Ferrand, I. N. R. A., \\ 63 - Saint-Genès-Champanelle
}

\section{RÉSUMÉ}

Les conséquences de la distribution à des vaches d'un composé minéral assurant dans la ration $0,2 \mathrm{mg}$ de sélénium par $\mathrm{kg}$ de matière sèche, sur la teneur en sélénium du lait de ces vaches et sur la teneur en cet élément des tissus des veaux recevant un tel lait, ont été examinées.

La teneur en sélénium du lait produit par les vaches était de l'ordre de celle des laits normaux.

Les veaux ayant reçu un lait enrichi en sélénium n'ont pas produit des carcasses risquant d'être toxiques pour le consommateur.

La prophylaxie de la myopathie des veaux par distribution à leurs mères de compléments minéraux enrichis en sélénium peut être réalisée sans danger pour les consommateurs dans la mesure où les teneurs en sélénium des mélanges minéraux sont garanties et contrôlées.

\section{INTRODUCTION}

I a myopathie du Veau en France est due à une teneur insuffisante des fourrages en sélénium (LAMAND, I970 b). Plusieurs méthodes de prophylaxie ont été proposées chez les ruminants par différents auteurs :

- la distribution de solutions de sélénite ou de séléniate de sodium aux jennes; (Sharmann et al., I959; HARTLEY et Grant, I96I ; OLDFIELD et al., I963, etc)

-- l'injection de sélénium aux mères et aux jeunes (OLDFIELD et al., I963; NELSON et al., I964, etc.).

- 1'administration de sélénium en mélange dans des comprimés d'oxyde de fer (KUCHEL et BUCKLEY, I969; HANDRECK et GODWIN, I970). 
La distribution aux vaches de composés minéraux contenant du sélénium est également efficace (LAMAND, I965).

La législation française et européenne interdit actuellement l'utilisation de cet élément dans les aliments. La toxicité éventuelle du sélénium à long terme n'est pas connue ; il importe donc, vis-à-vis du consommateur de lait ou de viande, de montrer toute la prudence souhaitable dans l'emploi de cet élément en élevage. Pour évaluer le bien-fondé de cette interdiction, nous avons voulu préciser les diverses conséquences d'une telle prophylaxie sur la composition du lait des mères d'une part, et, d'autre part, sur les teneurs en sélénium des différents tissus des veaux recevant un lait provenant de ces vaches.

\section{MATÉRIEL, ET MÉTHODES}

Dix vaches Frisonnes et ro vaches Montbéliardes ont reçu un mélange minéral dont la formule était la suivante :

Carbonate de calcium I 2 p. Ioo, phosphate bicalcique 48 p. Ioo, chlorure de sodium 23 p. Ioo, sulfate de magnésium, 8 p. Ioo, magnésie, 3 p. Ioo, oligo-éléments (Cu, $\mathrm{Co}, \mathrm{I}, \mathrm{Zn}, \mathrm{Mn}, \mathrm{Se}$ ) 6 p. Ioo.

Ce mélange renfermait $\mathrm{r} 7,5 \mathrm{mg}$ de sélénium par $\mathrm{kg}$, sous forme de sélénite de sodium. Distribué à raison de $\mathrm{I} 40 \mathrm{~g} / \mathrm{j}$ et par vache; il apportait $2,45 \mathrm{mg}$ de sélénium $/ \mathrm{j} / \mathrm{vache}$ et ramenait ainsi la ration de base des animaux à $0,2 \mathrm{mg}$ Se/kg MS environ.

Par ailleurs, en vue de fabriquer un aliment d'allaitement carencé, nous avons dosé le sélénium dans 35 laits de mélange, destinés à une laiterie, provenant de tournées de ramassage dans une aire géographique où sévit la Myopathie avec une fréquence très variable et où les veaux sont élevés avec du lait entier de l'exploitation. Les quatre stocks de lait les plus carencés ont été mélangés et séchés par procédé Spray sans avoir été préalablement écrémés.

- Un premier lot de ro veaux de race Frisonne a reçu cet aliment d'allaitement renfermant $0,03 \mathrm{mg} / \mathrm{kg}$ MS de sélénium.

- Un deuxième lot de 8 veaux, servant de témoin, a reçu ce même aliment contenant $0,4 \mathrm{mg}$ Se/kg MS grâce à la supplémentation de sélénite de sodium.

- Un troisième lot de 9 veaux de race Frisonne a été élevé dans les mêmes conditions avec un aliment d'allaitement dont la poudre de lait provenait d'une région indemne de myopathie. Cet aliment avait une teneur de o,o9 $\mathrm{mg}$ de Se/kg MS.

Pour le dosage du sélénium, les laits crus ont été dégraissés par centrifugation puis lyophilisés, les aliments d'allaitement ont été dégraissés à l'iso-octane pour éviter les risques d'explosion durant la minéralisation par voie humide. Le sélénium contenu dans les graisses est, en effet, négligeable (McCoNNEL, I963).

En fin d'expérience, nous avons déterminé, après lyophilisation, les teneurs en sélénium des tissus suivants : couche corticale du rein, lobule de Spiegel pour le foie, échantillon moyen de cour et muscle sternohyoïdien. Le sélénium a été dosé par une méthode dérivée de celle de WATKINson (LAMAND, 1969).

\section{RÉSULTATS}

Ires vaches recevant un supplément de sélénium dans leur ration ont produit un lait dont la teneur variait faiblement d'une race à l'autre (tabl. I). Pour les races Frisonne et Montbéliarde, les teneurs étaient respectivement de $0,15 \mathrm{I}$ et $0,12 \mathrm{I} \mathrm{mg} / \mathrm{kg}$ de MS, soit une teneur en sélénium très inférieure à celle de l'aliment supplémenté reçu par les veaux témoins.

Les laits de mélange provenant d'une région atteinte de myopathie renfermaient $0,058 \mathrm{mg} / \mathrm{kg} \mathrm{MS} \pm 0$, or $7(\bar{x} \pm \sigma)$ ou $0,0056 \mathrm{mg} /$ litre $\pm 0,002(\bar{x} \pm \sigma)$ avec des extrêmes de $0,027 \mathrm{mg} / \mathrm{kg}$ MS à $0,100 \mathrm{mg} / \mathrm{kg}$ MS. 
La teneur en sélénium des tissus des veaux supplémentés (tab1. 2) est environ le double de celle des veaux normaux, alors que le rapport de la teneur des laits de ces deux lots est de I à 4 . Le rapport de la teneur des tissus des veaux carencés à celle des veaux supplémentés est de I à 3 ou 4 alors que la teneur en sélénium des aliments correspondants est dans un rapport de I à I3.

Dans le lot carencé, 3 animaux sont morts de dégénérescence du myocarde, deux autres animaux ont montré à l'abattage des lésions cardiaques anciennes.

TABLEAU I

Teneur en sélénium du lait de vaches dont la ration était portée à $0,2 \mathrm{mg} / \mathrm{hg} M S$ de sélénium.

TABLE I

Selenium content of milk from cows receiving a diet containing $0,2 \mathrm{mg}$ Se/kg $\mathrm{DM}$

\begin{tabular}{|c|c|c|}
\hline & $\mathrm{mg} / \mathrm{kg}$ MS & mg/litre \\
\hline $\begin{array}{c}\text { Race Frisonne }(10) * \\
\text { Frisian Breed }\end{array}$ & $0,151+0,0 \div 6$ & $0,01143 \pm 0,0026$ \\
\hline $\begin{array}{c}\text { Race Montbéliarde }(10) * \\
\text { Montbéliarde Breed }\end{array}$ & $0,121 \pm 0,0 \geq 1$ & $0,012+40,0020$ \\
\hline \multirow{2}{*}{$\begin{array}{l}\text { Valeur de } t \\
t \text { value }\end{array}$} & $\begin{array}{c}2,86 \mathrm{P}<0,05 \\
(\lim 5 \%=2,101)\end{array}$ & $\begin{array}{c}1,9 \mathrm{P}>0,05 \\
(\lim .5 \%=2,101)\end{array}$ \\
\hline & mg/kg DM & 1. $\|_{\text {I }}$ mg/liter \\
\hline
\end{tabular}

(*) Nombre d'animaux.

Animals number.

\section{DISCUSSION}

Différents auteurs ont analysé des laits du commerce ou provenant de différents élevages. Ces résultats sont résumés dans le tableau 3.

Le lait des vaches ayant reçu une supplémentation normale en sélénium avait une teneur intermédiaire qui semble donc devoir être considérée comme normale et non dangereuse pour l'homme. Cette teneur est cependant supérieure à celle des laits de mélange d'une zone où la Myopathie sévit à l'état enzootique avec une fréquence variable. Dans cette zone, les laits renfermaient de 0,027 à $0,100 \mathrm{mg} / \mathrm{kg}$ MS de sélénium. Avec un lait renfermant o,090 $\mathrm{mg} / \mathrm{kg}$ MS de sélénium, nous n'avons pas observé de Myopathie. Si nous acceptons la limite de la carence à o, I mg/kg MS proposée pour les fourrages par MUTH et ArLAWAy (Ig63), les vaches ayant reçu une ration supérieure à $0,2 \mathrm{mg} / \mathrm{kg}$ ne devraient pas produire des veaux myopathiques.

La teneur en sélénium du lait a significativement influencé la teneur en sélénium des tissus des veaux ayant consommé ce lait. Le cour, le foie et les reins ont été nota- 
TABLEAU 2

Sélénium tissulaive des veaux ayant reçu différents laits (en $\mathrm{mg} / \mathrm{kg}$ )

TABIE 2

Selenium content of calves tissues receiving different milks $(\mathrm{mg} / \mathrm{kg})$

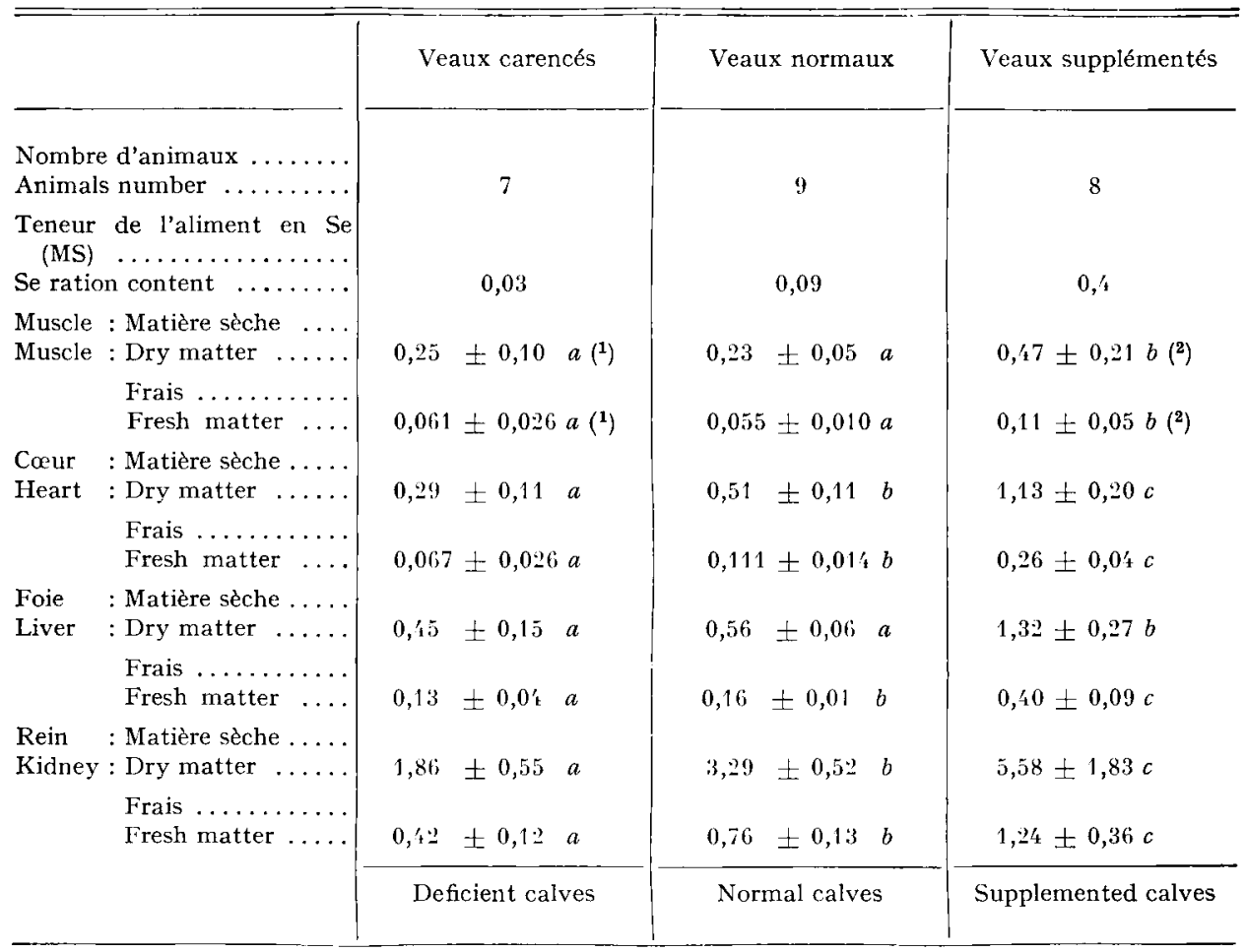

(1) Les moyennes d'une même ligne suivies de lettres différentes sont significativement différentes $(\mathrm{P}<0,01)$.

Means of the same line followed by different letters are significantly different.

(2) Cette moyenne est significativement différente des précédentes au seuil 5 p. $100(\mathrm{P}<0,05)$. This mean is different from the former at the 5 p. 100 level.

blement enrichis en sélénium alors que l'enrichissement du muscle s'est avéré moindre. MORRIS et LEVANDER (I970) ont prélevé de la viande dans un circuit commercial dans le Maryland et ont trouvé les teneurs de sélénium suivantes :

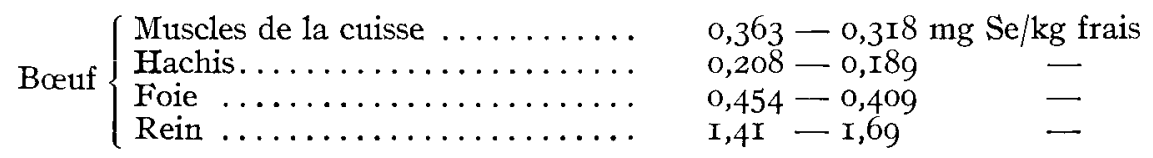

On notera que les veaux largement supplémentés en sélénium (lait à $0,4 \mathrm{mg} / \mathrm{kg}$ MS) n'ont pas produit une viande ni des abats toxiques, les teneurs en sélénium étant inférieures à celles rencontrées dans la viande de bœuf par MORRIs et LEVANDER. 
Les valeurs que nous avons obtenues sont également du même ordre que celles publiées par HIDIROGLov et al. (I967) pour des veaux normaux et indemnes de Myopathie, et sont voisines des résultats obtenus pour le Veau par Von OELSCHLÄGER et MENKE (I969), en Allemagne.

$$
\text { TABLEAU } 3
$$

Teneurs en sélénium des laits écrémés de différentes origines

TABLE 3

Selenium contents of skimmed milks of different origins

\begin{tabular}{|c|c|c|c|}
\hline Auteurs & $\begin{array}{l}\text { Origine du } \\
\text { prélèvement }\end{array}$ & $\begin{array}{c}\text { Moyenne } \\
\text { (mg/kg MS) }\end{array}$ & $\begin{array}{c}\text { Extrêmes } \\
(\mathrm{mg} / \mathrm{kg} \mathrm{MS})\end{array}$ \\
\hline 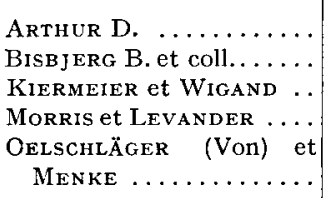 & $\begin{array}{l}\text { Canada } \\
\text { Danemark } \\
\text { Allemagne } \\
\text { États-Unis } \\
\text { Allemagne }\end{array}$ & 0,120 & $\begin{array}{l}0,100-0,170 \\
0,250-0,780 \\
0,080-0,152 \\
0,096-0,210\end{array}$ \\
\hline Authors & Country & $\begin{array}{c}\text { Mean } \\
\text { (mg/kg DM }\end{array}$ & $\begin{array}{c}\text { Extremes } \\
(\mathrm{mg} / \mathrm{kg} \mathrm{DM})\end{array}$ \\
\hline
\end{tabular}

La supplémentation des veaux par l'intermédiaire du complément minéral distribué aux mères, paraît donc réalisable sans présenter de danger pour les consommateurs du lait et des viandes des veaux.

Hidrogrou et al. (I97I) ont examiné les conséquences sur la teneur en sélénium des carcasses de la prophylaxie de la Myopathie par injection de sélénium à des bouvillons, des veaux ou des brebis. Ces auteurs concluent que cette prophylaxie est sans danger pour le consommateur à condition de respecter un délai d'attente entre l'injection et l'abattage des animaux. Or, ce délai peut parfois ne pas être respecté et des surdosages accidentels au moment de l'injection restent à craindre. Il n'en reste pas moins que ce type de prophylaxie est le seul pratiqué en France actuellement.

Les injections, outre le prix d'achat du sélénium injectable, sont relativement coûteuses en main-d'œuvre et ne sont réalisées dans de bonnes conditions qu'après un effort soutenu des organismes de développement de l'élevage.

La distribution de composés minéraux supplémentés en sélénium permettrait une extension plus large de la prophylaxie de la Myopathie sans augmenter notablement le prix des mélanges destinés à apporter les oligo-éléments. Elle éviterait d'éventuels surdosages. A titre expérimental, nous avons fait fabriquer de tels mélanges dont l'homogénéité s'est avérée bonne. La variance d'un échantillon à l'autre n'était pas significativement différente de l'erreur due au dosage. Le dosage du sélénium dans les mélanges minéraux ne pose pas de difficulté majeure et pourrait être réalisé dans des contrôles de routine (LAMAND, I969).

Chez le Mouton, Paulson et al. (I968), aux États-Unis, ont essayé de distribuer ad libitum un complément minéral enrichi en sélénium. Ils n'ont pas observé d'accu- 
mulation de l'élément dans les carcasses des agneaux. La teneur en sélénium des tissus de ces animaux était de l'ordre de celles rencontrées chez des agneaux recevant des rations non carencées provenant de diverses régions des États-Unis.

L'apport de sélénium ainsi proposé par ces auteurs reste inférieur à la libération de sélénium obtenue dans le rumen à partir de comprimés de fer et sélénium essayés par Kucher et BuckleEy (I969) et Handreck et Godwin (I970) chez la Brebis. Ces auteurs ont, en effet, obtenu, avec les comprimés essayés, une libération quotidienne d'environ I mg de sélénium. Cette dose journalière est probablement supérieure aux besoins, bien que les carcasses obtentues aient une teneur normale en sélénium.

En conclusion, la prophylaxie de la Myopathie par la distribution de compléments minéraux enrichis en sélénium semble réalisable et sans danger pour les consommateurs du lait des vaches traitées et des viandes de veaux élevés avec ce lait. Ces mélanges doivent avoir une teneur garantie en sélénium et homogène. Ce type de prophylaxie est plus facilement contrôlable tout en étant moins coûteuse à réaliser et à généraliser que les injections actuellement pratiquées, susceptibles de présenter des inconvénients pour le consommateur.

Reçu pour publication en aồt 1971.

\section{SUMMARY}

POSSIBILITY OF PREVENTING MUSCULAR DYSTROPHY IN THE CALF BY ADDING SELENIUM TO THE MINERAL SUPPLEMENTS OF THE DAMS

In the present study, the consequences, for the meat consumer, of preventing muscular dystrophy in the calf by distributing selenium enriched mineral supplements to the cows, were investigated.

Ten Frisian cows and ten Montbéliard cows received selenium enriched diets $(0.2 \mathrm{mg} / \mathrm{kg}$ of DM) owing to a mineral supplement. The cows produced milk containing O.I5I and o.I2 I mg selenium per $\mathrm{kg}$ of DM. This content can be considered as normal.

In addition, ten calves received a selenium deficient milk replacer $(0.03 \mathrm{mg} / \mathrm{kg} \mathrm{DM})$. The muscle, heart, liver and kidney contained $0.25,0.29,0.45$ and $\mathrm{r} .86 \mathrm{mg}$ selenium respectively per $\mathrm{kg}$ dry matter.

Nine calves received a normal milk replacer $(0.09 \mathrm{mg} \mathrm{Se} / \mathrm{kg}$ of DM) and the selenium contents of the muscle, heart, liver and kidney respectively were the following : $0.23,0.5 \mathrm{I}, 0.56$ and $3.29 \mathrm{mg} /$ kg DM.

Eight calves received a selenium enriched milk replacer $(0.4 \mathrm{mg} / \mathrm{Se} / \mathrm{kg}$ DM) resulting in the following selenium contents in the muscle, heart, liver and kidney respectively : 0.47, I.I3, I.32 and $5.5^{8}$ per $\mathrm{kg}$ dry matter.

Selenium supplementation of the calves' diets did not result in production of meat and offals being unfitted for human feeding because of a high selenium content.

It may be concluded that muscular dystrophy in calves can be prevented by distributing selenium enriched mineral complements to the dams and that this supplementation can be practised without any danger for the consumer, provided that the selenium content of the mineral mixtures is guaranteed and controled. 


\section{RÉFÉRENCES BIBLIOGRAPHIQUES}

Arthur D., I971. Selenium content of some feed ingredients available in Canada. Can. J. Anim. Sci., $51,71-74$.

Bisbjerg B., Jocilumsen P, Rasbech N. O., I97o. Selenium in organs, milk and fodder of the cow. Nord. Vet. Med., 22, 532-535.

McConnel K. P., r963. Metabolism of selenium in the mammalian organism. J. Agric. Food Chem., 11, $385-388$.

HANDRECK $\quad$ K. A., GODWIN K. O., I970. Distribution in the sheep of selenium derived from ${ }^{57}$ Se labelled ruminal pellets. Aust. J. agric. Res., 21, 7I-84.

Hartiey W. J., Grant A. B., rg6r. A review of selenium responsive diseases of New Zcaland livestock. Fed. Proc., 20,679.

Hidiroglou M., Jenkins K. J., Carson R. B., Brossari G. A., I967. Selenium and coenzyme $Q_{10}$ levels in the tissues of dystrophic and healthy calves. Can. J. Physiol. Pharmac., 45, 568-569.

Hidiroglou M., Jenkins K. J., Hoffman I., i97i. Teneurs en sélénium dans les tissus des ruminants. Ann. Biol. anim. Bioch. Biophys., 11, 695-704.

Kiermeier T., Wigand W., I969. Selengehalte von Milch und Milchpulver. Z. Lebensmittel Unters. Forschung, 139, 205-2II.

KUсHжL R, E., BUCKLeY R. A., r969. The provision of selenium to sheep by means of heavy pellets. A ust. J. agric. Res., 20, ro99-1 107.

LAMAND M., I965. Syndrome Myopathie-Dyspnée : influence de la prophylaxie par le sélénium sur la composition biochimique du plasma chez le Veau. Ann. Biol. anim. Bioch. Biophys., 5, 309-3I5.

LAmand M., r 969 . Dosage du sélénium dans les produits biologiques et mélanges minéraux alimentaires. Ann. Fals. Exp. Chim. (1), 4-I2.

Lamand M., I97o $a$. Lésions biochimiques de la Myopathie du veatu par carence en sélénium. $C . R$. Acad. Sci. Série D, 270, 4I 7-420.

Lamand M., I97o b. Expansion de la Myopathie en France en liaison avec la carence en sélénium des fourrages. C. R. Séances Hebdo. Acad. Agric. de France, 56, 604-610.

Morris V. C., Levander O. A., I970. Selenium content of foods. J. Nutr., 100, 1383-1 388.

Muti O. H., Allaway W. H., I963. The relationship of white muscle disease to the distribution of naturally occuring selenium. J. amer. Vet. Med. Ass., 142, I379.

Nelson I. C., Hidiroglou M., Hamilton H. A., I964. The effect of prophylactic treatment of pregnant beef cows on the inciderice of nutritional muscular dystrophy. A field trial. Can. Vet. J., 5, $268-273$.

Orischläger Von W., MENKE K. H., I969. Uber Selengchalte pflanzlicher, tierischer und anderer Stoffe. 2. Mitteilung. Selen und Schwefelgehalte in Nahrungsmitteln. Zeitschr. Ernährungswissenschaft, 9, 2 I6-222.

OldFifld J. E., Schubert J. R., Muth O. H., I963. Implications of selenium in large animal nutrition. A gr. Food Chem., 11, 388 .

Paulson G. D., Broderick G. A., Baumann C. A., Pope A. L., ig68. liffect of feeding sheep selenimm fortified trace mineralized salt : effect of tocopherol. J. Anim. Sci., 27, 195-202.

Sharmann G. A. M., Blaxter K. L., Wilson IR. S., I959. Prevention of enzootic muscular dystrophy by selenium administration. Vet. Rec., 71, 536. 